\title{
Hybrid Model Predictive Control as a LFC solution in Hydropower Plants
}

\author{
Emerson Donaisky ${ }^{1, a}$, Gustavo H. C. Oliveira ${ }^{2}$, Eduardo A. P. Santos ${ }^{1}$, \\ Gideon V. Leandro ${ }^{2}$, Adinã M. Pena ${ }^{3}$, João A. Souza ${ }^{4}$ \\ 1 Polytechnic School, Pontifical Catholic University of Paraná, Curitiba, Brazil \\ 2 Electrical Engineering Department, Federal University of Paraná, Curitiba, Brazil \\ CEMIG-GT, Belo Horizonte, Brazil \\ 4 COPI Control, Belo Horizonte, Brazil
}

\begin{abstract}
For Electric Power System safety and stable operation, planning and analysis by using simulation environments are necessary. An important point for frequency stability analysis is, on one hand, an adequate representation of Load-Frequency Control (LFC) loops and, on the other hand, the design of advanced control strategies to deal with the power system dynamic complexity. Therefore, in this paper we propose to represent the group turbine/penstock, found in hydropower plants, in a Piecewise Affine (PWA) modelling structure. Based on such modelling, we also propose the use of a Hybrid Model Predictive algorithm to be use as a control law in LFC loops. Among the advantages of this PWA representation is the use of this model in the controller algorithm, thereby improving the Load-Frequency Control performance. Simulation results, on a $200 \mathrm{MW}$ hydropower plant compares the performance of predictive control strategy presented with the classical PID control strategy in an isolated condition.
\end{abstract}

\section{Introduction}

The stability of Electric Power System (EPS) can be defined as the ability to maintain frequency, voltage and power balance in the nominal conditions, even after being subjected to some disturbance [1]. Due to continuous changes on power demand and also the system disturbances the EPS is submitted, the EPS operating point is continuously changing so as its models. This feature makes challenging the development of control laws that assures a stable operation and the specified performance to restore the nominal conditions.

Many controllers are involved in the electricity generation to guarantee power system stability. In this paper, we are interested on control balance between the power generation and demand, where the frequency is an important indicator [2]. Therefore, there is a controller responsible for manipulating the power generation in order to comply it with the network power demand and also for ensuring that the frequency delivered to the grid is at its nominal value. It is so called Load-Frequency Control (LFC) and it is becoming increasingly important due to the growing in complexity and interconnection of the power system [3].

This LFC loop involves several nonlinear devices. The modelling of these devices and the analysis of the influence of nonlinearities in the LFC loop have been reported in the literature since the $60 \mathrm{~s}$, for instance [4]. One of them is the group turbine/generator. An appropriate mathematical representation of such group allows improving the controller tuning in such a way that the performance of the frequency control loop is augmented.

In $[5,6,7]$ are presented several detailed hydro turbines models for using in power system stability studies, ranging from simple to complex models, with linear dynamics, non-linear dynamics, even taking into account the elasticity of the penstock. In the present work, we recall the idea of using linear models with time varying parameters aiming to adequately representing several operating points [8,9]. Also, two contributions are described. First, the linear time varying model for the group turbine/penstock is translated to a Piecewise Affine (PWA) model structure, which is a class of switched hybrid systems, that is, systems that have a relationship between dynamic systems (ruled by time) and discrete event systems (ruled by logical events) [10]. Second, based on the proposed model, a Hybrid Model Based Predictive Control is presented as a control solution for LFC in hydropower plants.

The paper is structured as follows. Section 2 contains a mathematical model for LFC loop in hydropower plants. In Section 3, the main concepts relating to the PWA system are presented and the PWA representation for the LFC loop is proposed. In Section 4, a Hybrid Predictive Control algorithm based on the PWA system representation is presented. Section 5 discusses an example consisting of a $200 \mathrm{MW}$ generator speed control loop in a hydropower plant. Finally, in Section 6, the conclusions are stated.

\footnotetext{
${ }^{\mathrm{a}}$ Corresponding author: emerson.donaisky@pucpr.br
} 


\section{Load-Frequency Control Mathematical Models}

The mathematical model for LFC in Hydropower Plants is depicted in Fig. 1 [7]. It consists of the control law, the hydraulic amplifier, responsible for transforming the electrical control signal into a power signal capable to move the turbine components, the group turbine/penstock and the synchronous machine, which generates electricity. Such representation is consistent with single area control, that is, a power plant operating in island condition.

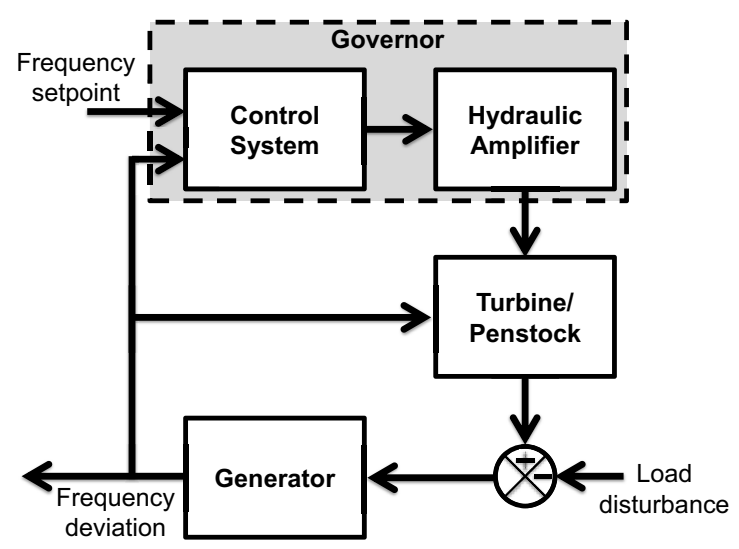

Figure 1. Block diagram of the LFC loop in a HPP.

The hydraulic amplifier is considered here a second order system with time constant $T_{Z}$, according to Eq. 1 . The generator is represented by a first order system with mechanical starting time $T_{M}$ and machine electrical damping coefficient $D$, according to Eq. 2 [11].

$$
\begin{aligned}
& H(s)=\frac{1}{\left(T_{Z} s+1\right)^{2}} \\
& G(s)=\frac{1}{T_{M} s+D}
\end{aligned}
$$

Fig. 2 reproduces, in a block diagram, the linear model described in [12] for representing the group turbine/penstock. Here, we are assuming that each parameter of such model varies on time depending on the active power generated by the power plant.

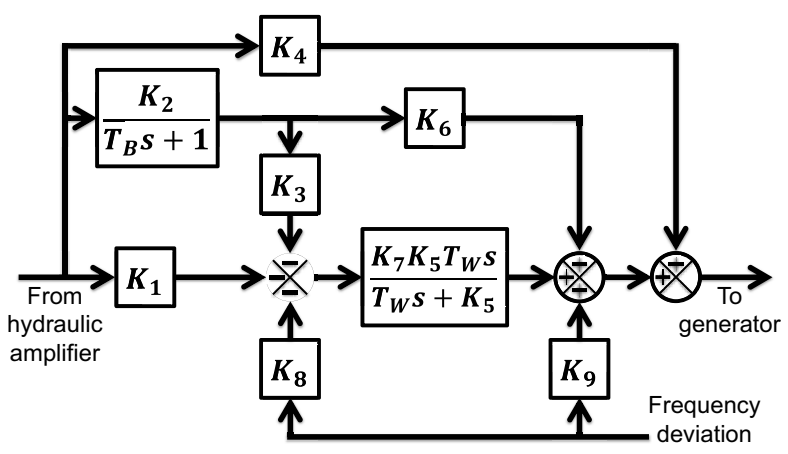

Figure 2. Block diagram of the group turbine/penstock.

The final model for the LFC loop components, from the hydraulic amplifier output $U(s)$, load disturbance $\mathrm{D}(\mathrm{s})$ representing the electrical power demand, to the generator frequency output $\mathrm{Y}(\mathrm{s})$ can be rewritten as follows:

$$
\begin{gathered}
\operatorname{num}(s)=T_{B} T_{W}\left(K_{4}-K_{1} K_{5} K_{7}\right) s^{2}+\left(T_{B} K_{4} K_{5}+\right. \\
\left.T_{W}\left(K_{4}+K_{2} K_{6}-K_{1} K_{5} K_{7}-K_{2} K_{3} K_{5} K_{7}\right)\right) s+ \\
K_{5}\left(K_{4}+K_{2} K_{6}\right) \\
\operatorname{den}(s)=T_{B} T_{W} T_{M} s^{3}+\left(T_{M}\left(T_{W}+T_{B} K_{5}\right)+\right. \\
\left.T_{B} T_{W}\left(D-K_{9}+K_{5} K_{7} K_{8}\right)\right) s^{2}+\left(K_{5} T_{M}+T_{B}\left(K_{5} D-\right.\right. \\
\left.\left.K_{5} K_{9}\right)+T_{W}\left(D-K_{9}+K_{5} K_{7} K_{8}\right)\right) s+K_{5}\left(D-K_{9}\right) \\
Y(s)=\frac{n u m(s)}{\operatorname{den}(s)} U(s)+ \\
+\frac{-T_{W} s-K_{5}}{T_{M} T_{W} s^{2}+\left(T_{W}\left(D-K_{9}+K_{5} K_{7} K_{8}\right)+K_{5} T_{M}\right) s+K_{5}\left(D-K_{9}\right)} D(s)
\end{gathered}
$$

\section{Model Representation as a PWA System}

Continuous linear dynamical systems are usually represented by transfer functions. Moreover, some systems have dynamic given by events and not by the time. Such systems are called discrete event systems. Hybrid systems are defined when there is a relationship between continuous dynamical systems and discrete event systems. Among the hybrid systems, we can find the Piecewise Affine (PWA) systems [10]. In PWA systems, there are regions defined by operating points, and for each region, there is associated a linear system (or affine). It is specified by dividing the system state space into polyhedral regions and associating to each of these regions a different linear (or affine) state equation. Eq. 4 gives this representation.

$$
F_{P W A}=\left\{\begin{array}{c}
x(k+1)=A_{i} x(k)+B_{i} u(k)+f_{i} \\
y(k)=C_{i} x(k)+D_{i} u(k)+g_{i} \\
\text { for }[x(k) u(k)]^{T} \in \Omega_{i}
\end{array}\right.
$$

where $x$ is the state vector, $u$ is the input vector, $y$ is the output and $\left\{\Omega_{i}\right\}_{i=1}^{m}$ are the $m$ polyhedral regions of the state space defined by the system inequalities $\left\{H_{i}[x(k) \quad u(k) \quad 1]^{T} \preccurlyeq 0\right\} . A_{i}, B_{i}, f_{i}, C_{i}, D_{i}, g_{i}$ and $H_{i}$ are real constant matrices with appropriate dimensions and $k$ is the discrete time. Although the notation may be unusual, in this equation the symbol $\leqslant$ denotes a vector of which the elements can be the symbols $\leq$ and $<$ to avoid overlap among the regions $\Omega_{i}$ over common boundaries.

\subsection{PWA Model for the LFC Loop Components}

We propose to rewrite the LFC model in terms of PWA model in such a way that only one model can characterize the whole turbine/penstock model behaviour in several operating points. We define a grid of power generation values, consistent with different power system operation values. Each part of the grid is associated with a linear model with constant parameters and regions in the state space defined by $H_{i}$. The set of all grids defines the LFC PWA model following Eq. 4. 
The continuous time PWA model for the group turbine/penstock and generator, derived from Eq. 3, is given by Eq. 5 , where $x(t)$ is the state vector, $u(t)$ is the vector of inputs (hydraulic amplifier output and load disturbance) and $y(t)$ is the generator frequency output.

$$
\begin{aligned}
& \left\{\begin{array}{c}
\dot{x}(t)=\left[\begin{array}{cc}
A_{T P G} & \mathbf{0} \\
\mathbf{0} & A_{L D}
\end{array}\right] x(t)+\left[\begin{array}{cc}
B_{T P G} & \mathbf{0} \\
\mathbf{0} & B_{L D}
\end{array}\right] u(t) \\
y(t)=\left[\begin{array}{ll}
C_{T P G} & C_{L D}
\end{array}\right] x(t)
\end{array}\right. \\
& F_{T P G} \equiv\left\{\begin{array}{c}
A_{T P G}=\left[\begin{array}{ccc}
0 & 1 & 0 \\
0 & 0 & 1 \\
a_{1} & a_{2} & a_{3}
\end{array}\right], B_{T P G}=\left[\begin{array}{l}
0 \\
0 \\
1
\end{array}\right], \\
C_{T P G}=\left[\begin{array}{lll}
c_{1} & c_{2} & c_{3}
\end{array}\right]
\end{array}\right. \\
& F_{L D} \equiv\left\{\begin{array}{c}
A_{L D}=\left[\begin{array}{cc}
0 & 1 \\
a_{4} & a_{5}
\end{array}\right], B_{L D}=\left[\begin{array}{l}
0 \\
1
\end{array}\right], \\
C_{L D}=\left[\begin{array}{ll}
c_{4} & c_{5}
\end{array}\right]
\end{array}\right. \\
& a_{1}=\frac{K_{5}\left(K_{9}-D\right)}{T_{B} T_{W} T_{M}} \\
& a_{2}=\frac{T_{B} K_{5}\left(K_{9}-D\right)+T_{W}\left(K_{9}-D-K_{5} K_{7} K_{8}\right)-T_{M} K_{5}}{T_{B} T_{W} T_{M}} \\
& a_{3}=\frac{T_{B} T_{W}\left(K_{9}-D-K_{5} K_{7} K_{8}\right)-T_{M}\left(T_{W}-T_{B} K_{5}\right)}{T_{B} T_{W} T_{M}} \\
& a_{4}=\frac{K_{5}\left(K_{9}-D\right)}{T_{W} T_{M}} \\
& a_{5}=\frac{T_{W}\left(K_{9}-D-K_{5} K_{7} K_{8}\right)-T_{M} K_{5}}{T_{W} T_{M}} \\
& c_{1}=\frac{K_{5}\left(K_{4}+K_{2} K_{6}\right)}{T_{B} T_{W} T_{M}} \\
& c_{2}=\frac{T_{B} K_{4} K_{5}+T_{W}\left(K_{4}+K_{2} K_{6}-K_{1} K_{5} K_{7}-K_{2} K_{3} K_{5} K_{7}\right)}{T_{B} T_{W} T_{M}} \\
& c_{3}=\frac{K_{4}-K_{1} K_{5} K_{7}}{T_{M}} \\
& c_{4}=\frac{-K_{5}}{T_{W} T_{M}} \\
& c_{5}=\frac{-T_{W}}{T_{W} T_{M}}
\end{aligned}
$$

The final model for the LFC loop components, from the control output and load disturbance, to the generator frequency output can be written in continuous time PWA structure as follows:

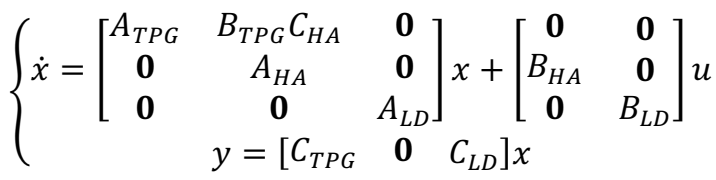

$$
\begin{aligned}
& F_{H A} \equiv\left\{\begin{array}{c}
A_{H A}=\left[\begin{array}{cc}
0 & 1 \\
-T_{Z}^{-2} & -2 T_{Z}^{-1}
\end{array}\right], B_{H A}=\left[\begin{array}{l}
0 \\
1
\end{array}\right], \\
C_{H A}=\left[T_{Z}^{-2} \quad 0\right]
\end{array}\right.
\end{aligned}
$$

The discrete time model for the LFC loop can be obtained assuming a sample time $\Delta t$ and using some methodology for the state space discretization [13]. For each part of the grid, the matrix parameters from the model are changed, originating the PWA model, structured as Eq. 4.

\section{Hybrid Model Predictive Control for PWA Systems}

Model Based Predictive Control (MBPC) is defined by the following steps: $i$ ) a model is used to compute the process output predictions; ii) a cost function related to the performance of the closed-loop system is defined; iii) this cost function is minimized over the future control signals set; $i v$ ) the first of these optimal control signals is applied to the process, i.e., the rolling horizon strategy.

The coast function of MBPC is given by Eq. 7, where $N_{y}$ and $N_{u}$ define the prediction and control horizons, respectively; $\mu$ is a weighting factor in the prediction error between the output signal $\hat{y}$ and the set-point signal $w ; \lambda$ is a weighting factor in the control signal; and $\Delta u(k+j \mid k)$ is the control variation signal in time $k+j$ calculated at time $k$.

$$
\begin{aligned}
& J(k)=\sum_{j=1}^{N_{y}} \mu(j)\|\hat{y}(k+j \mid k)-w(k+j)\|^{2}+ \\
& \sum_{j=0}^{N_{u}-1} \lambda(j)\|\Delta u(k+j \mid k)\|^{2}
\end{aligned}
$$

The control law is obtained by minimizing the cost function in relation to future variations in control signal $\Delta u(\cdot)$ according to Eq. 8 . The process is subject to constraints on control signal, according to Eq. 8. Since output predictions are obtained by means of a PWA model, we have the so called Hybrid Model Predictive Control (HMPC) [14]. Such control law is defined by the following nonlinear programming problem, with nonlinear constraints, where $\theta_{i}$ are the parameters for the PWA model.

$$
\begin{aligned}
& \min _{\Delta u(k \mid k), \cdots, \Delta u\left(k+N_{u}-1 \mid k\right)} J(k) \\
& \text { subject to: } \\
& \Delta u(k+j \mid k)=0 \quad \forall j=N_{u}, \cdots, N_{y} \\
& 0 \leq u(k+j-1) \leq 1 \quad \forall j=1, \cdots, N_{u} \\
& \hat{y}(k \mid k)=F_{P W A}\left(u(k), \theta_{i}\right) \quad \forall i=1, \cdots, m
\end{aligned}
$$

The optimal set of future control signals can be obtained using a mixed integer programming algorithm or optimization algorithms and the optimal control signal is calculated as follows:

$$
u(k)=u(k-1)+\Delta u(k \mid k)
$$

\section{Simulation Results}

To illustrate the operation of the proposed LFC loop representation as a PWA system, in this work is used a hydraulic turbine with $200 \mathrm{MW}$ capacity, equivalent to two generating units at the HPP Mactaquac Generating Station of the New Brunswick Electric Power Commission $[8,12]$. The turbine parameters for three distinct operating points (denominated T1, T2 and T3, as presented in [8]) are provided in Table 1. The other LFC loop time unvarying parameters are given in Table 2 . It is considered that the HPP was operating in islanded condition, that is, disconected from the EPS and supplying the load demand alone. 
Table 1. Turbine/Penstock coefficients.

\begin{tabular}{|c|c|c|c|}
\hline $\begin{array}{c}\text { Operating } \\
\text { Point (pu) }\end{array}$ & $\begin{array}{c}\text { T1 = 22.5 } \\
\text { MW }\end{array}$ & $\begin{array}{c}\text { T2 }=84.3 \\
\text { MW }\end{array}$ & $\begin{array}{c}\text { T3 }=113 \\
\text { MW }\end{array}$ \\
\hline$K_{1}$ & 0.80 & 0.40 & 0.38 \\
\hline$K_{2}$ & 0.0 & 2.3 & 1.0 \\
\hline$K_{3}$ & 0.00 & 0.30 & 0.69 \\
\hline$K_{4}$ & 0.88 & 0.90 & 0.34 \\
\hline$K_{5}$ & 0.06 & 0.20 & 0.24 \\
\hline$K_{6}$ & 0.00 & 0.50 & 0.52 \\
\hline$K_{7}$ & 0.4 & 1.2 & 1.5 \\
\hline$K_{8}$ & 0.13 & 0.38 & 0.62 \\
\hline$K_{9}$ & -0.39 & -0.86 & -0.75 \\
\hline
\end{tabular}

Table 2. Hydraulic amplifier, Turbine/Penstock and Generator parameters.

\begin{tabular}{|c|c|}
\hline $\boldsymbol{T}_{B}$ (seconds) & 3.5 \\
\hline $\boldsymbol{T}_{W}$ (seconds) & 2.35 \\
\hline $\boldsymbol{T}_{Z}$ (seconds) & 0.15 \\
\hline $\boldsymbol{T}_{M}$ (seconds) & 10.25 \\
\hline $\boldsymbol{D}$ & 0.8 \\
\hline
\end{tabular}

Using the sampling time $\Delta t=1.0$ seconds, this system, represented as a PWA structure, has $m=3$ polyhedral regions, representing the three operating points, which are equivalent to loads (per unit) of $0<\mathrm{T} 1<0.1125 \quad, \quad 0.1125 \leq \mathrm{T} 2 \leq 0.4215 \quad$ and $0.4215<\mathrm{T} 3<1$, and this points are used to construct the coefficients of matrix $H_{i}$, as follows:

$$
\begin{aligned}
& H_{1}=\left[\begin{array}{llr}
\mathbf{0}_{1 \times 8} & 1 & -0.1125
\end{array}\right] \\
& H_{2}=\left[\begin{array}{llr}
\mathbf{0}_{1 \times 8} & -1 & 0.1125 \\
\mathbf{0}_{1 \times 8} & 1 & -0.4215
\end{array}\right] \\
& H_{3}=\left[\begin{array}{lll}
\mathbf{0}_{1 \times 8} & -1 & 0.4215
\end{array}\right]
\end{aligned}
$$

It has been compared two control strategies, that are: $i$ ) the PID control strategy, where the Control System of Fig. 1 is replaced by Eq. 11 , with parameters $K_{p}=1.43$, $K_{i}=0.21$ and $K_{d}=0.54$; and the proposed predictive control strategy, with parameters $N_{y}=50, N_{u}=1$, $\mu=1$ and $\lambda=0$. The PID tuning was made for the worst operating point, and good response for the others operating points, and the HMPC tuning uses prediction horizon covering all system dynamics.

$$
C(s)=K_{p}+\frac{K_{i}}{s}+K_{d} s
$$

The test consisted basically of applying step load disturbances, which represent severe disturbances in HPP, as shown in Fig. 3. The result of the comparison between these two control strategies is shown in Figs. 4 and 5.

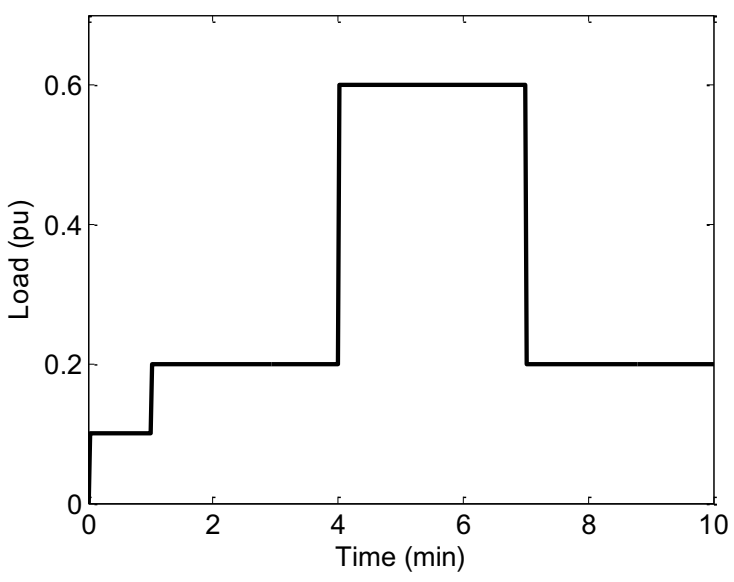

Figure 3. Step load disturbances.

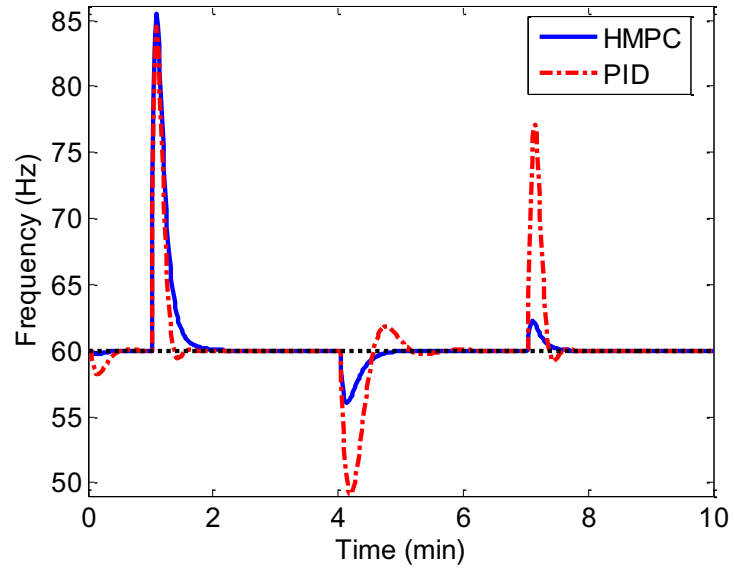

Figure 4. Frequency for PID and HMPC controllers.

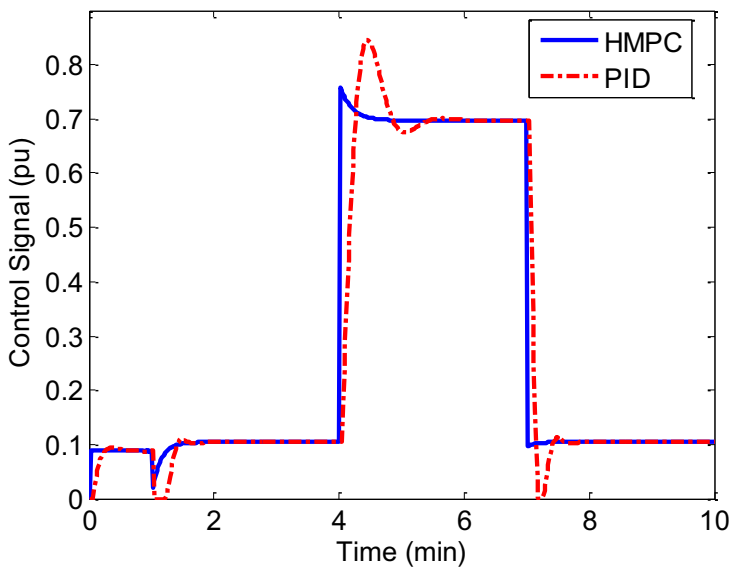

Figure 5. Control signal for PID and HMPC controllers.

As can be seen in Figs. 4 and 5, it is noted that both control strategies present a large excursion of the frequency in the machine output. This fact is caused by large load disturbances applied to the HPP, that must satisfy the demand alone. However, it is observed that the HMPC was able to restore the frequency at its nominal value in a shorter time. 


\section{Conclusion}

In this work, a new proposal for LFC law for Hydropower Plants is described. The aim is to improve frequency power system stability and closed loop performance in restoring the nominal conditions. Two points characterize the ideas discussed here. First, it is to relate the linear time varying parameter turbine/penstock model with a PWA model, which is a class of Hybrid System. Second, based on this model, a new Hybrid Model Predictive Control is described. Simulations results, on a $200 \mathrm{MW}$ hydropower plant model, operating in isolated condition, is conducted and, compared with classic PID control strategy, have shown an improved performance.

\section{References}

1. P. Kundur, J. Paserba, V. Ajjarapu, G. Andersson, A. Bose, C. Canizares, N. Hatziargyriou, D. Hill, A. Stankovic, C. Taylor, T. Van Cutsem, V. Vittal, IEEE Transactions on Power Systems 19, 1387-1401 (2004)

2. H. Bevrani, Robust power system frequency control 2, 391 (2014)

3. S.K. Pandey, S.R. Mohanty, N. Kishor, Renewable and Sustainable Energy Reviews 25, 318-334 (2013)
4. H.E. Lokay, R.L. Bolger, IEEE Transactions on Power Apparatus and Systems 84, 933-942 (1965)

5. I.C. Report, IEEE Transactions on Power Apparatus and Systems PAS-92, 1904-1915 (1973)

6. W.G. Report, IEEE Transactions on Power Systems 7, 167-179 (1992)

7. N. Kishor, R.P. Saini, S.P. Singh, Renewable and Sustainable Energy Reviews 11, 776-796 (2007)

8. K. Natarajan, IEEE Transactions on Energy Conversion 20, 661-667 (2005)

9. A. Izena, H. Kihara, T. Shimojo, K. Hirayama, N. Furukawa, T. Kageyama, T. Goto, C. Okamura, IEEE Power Engineering Society General Meeting, 7 (2006)

10. W.P.M.H. Heemels, D. Lehmann, J. Lunze, B. De Schutter, Handbook of Hybrid Systems Control: Theory, Tools, Applications 1, 3-30 (2009)

11. P. Kundur, N.J. Balu, M.G. Lauby, Power system stability and control 1, 1200 (1994)

12. D.T. Phi, E.J. Bourque, D.H. Thorne, E.F. Hill, IEEE Transactions on Power Apparatus and Systems PAS100, 3203-3212 (1981)

13. K.J. Astrom, B. Wittenmark, Computer-Controlled Systems: Theory and Design 3, 555 (1996)

14. E.F. Camacho, D.R. Ramirez, D. Limon, D. Muñoz de la Peña, T. Alamo, Annual Reviews in Control 34, 21-31 (2010) 